\title{
Термодинамическое описание осцилляций намагниченности кремниевой наноструктуры в слабых полях при комнатной температуре. Плотность состояний
}

\author{
(C) В.В. Романов ${ }^{1}$, В.А. Кожевников ${ }^{1}$, Н.Т. Баграев ${ }^{1,2, \text { ฯ }}$ \\ ${ }^{1}$ Санкт-Петербургский политехнический университет Петра Великого, \\ 195251 Санкт-Петербург, Россия \\ ${ }^{2}$ Физико-технический институт им. А.Ф. Иоффре Российской академии наук, \\ 194021 Санкт-Петербург, Росссия \\ ฯ E-mail:Bagraev@mail.ioffe.ru
}

Поступила в Редакцию 19 августа 2019 г.

В окончательной редакции 23 августа 2019 г.

Принята к публикации 23 августа 2019 г.

Наблюдение осцилляций де Гааза-ван Альфена при исследовании кремниевой наноструктуры при комнатной температуре в слабых магнитных полях делает возможным использование термодинамических соотношений для вычисления плотности состояний на уровне Ферми при критических значениях напряженности внешнего магнитного поля для целочисленных факторов заполнения.

Ключевые слова: намагниченность, кремниевый наносандвич, плотность состояний, эффект де Гааза-ван Альфена, равновесная термодинамика.

DOI: 10.21883/FTP.2019.12.48621.9242

\section{1. Введение}

В данной работе мы приводим термодинамическое описание осцилляций намагниченности двумерной кремниевой наноструктуры, технология приготовления которой позволила реализовать критерий сильного поля в слабых магнитных полях при комнатной температуpe $[1,2]$.

Выполненные ранее экспериментальные исследования дали основания идентифицировать наблюдаемые осцилляции намагниченности как эффект де Гааза-ван Альфена [1-3], что позволяет использовать термодинамические соотношения для получения важной информации о плотности состояний (DOS), являющейся важнейшей характеристикой энергетического спектра полупроводниковой структуры. В настоящем исследовании двумерная термодинамическая плотность состояний на уровне Ферми определялась из зависимости намагниченности образца от напряженности внешнего магнитного поля при достижении исследуемой системой состояния равновесия.

С этой целью в данной работе были проведены измерения намагниченности кремниевой сандвич-структуры с минимальной доступной в эксперименте ,пошаговой“ разверткой по магнитному полю (1Э), при которой удается наблюдать разрешенные осцилляции в эффекте де Гааза-ван Альфена до значений фактора заполнения $v=12$ включительно.

\section{2. Эксперимент}

Исследуемая кремниевая сандвич-структура представляет собой сверхузкую, 2 нм, квантовую яму ( $\mathrm{Si}-\mathrm{QW}$ ) $p$-типа проводимости, ограниченную $\delta$-барьерами, сильно легированными бором, на поверхности монокристаллического $\mathrm{Si}(100)$ n-типа проводимости [1] (рис. 1,a). Ограничение движения носителей $\delta$-барьерами, состоящими из дипольных центров бора с отрицательной корреляционной энергий, позволило реализовать такие качества структуры, как низкая эффективная масса носителей и сильное подавление электрон-электронного взаимодействия, что определило возможность наблюдения макроскопических квантовых эффектов в магнитных полях при комнатной температуре [1-3] (рис. 1, $b$ и $c$ ).

Отметим, что в исследуемом образце наблюдаются хорошо разрешенный квантовый эффект Холла (КЭХ) при температуре $T=77 \mathrm{~K}$ и осцилляции де Гааза-ван Альфена при комнатной температуре [1]. Необходимая для дальнейших вычислений двумерная плотность носителей, $3 \cdot 10^{13} \mathrm{M}^{-2}$, была найдена из холловских измерений [1].

Изучение магнитных свойств кремниевой наноструктуры проводилось путем анализа полевых зависимостей намагниченности образцов, измеренных методом Фарадея при комнатной температуре в интервале напряженностей магнитного поля от $H=100$ до 800 Э с дискретной разверткой внешнего магнитного поля с шагом по полю 1 Э на установке, созданной на базе спектрометра MGD 312 FG, в автоматическом режиме. Калибровка установки проводилась с помощью эталонного образца, в качестве которого использовался монокристалл магнитно-чистого фосфида индия с восприимчивостью $\chi=-313 \cdot 10^{-9} \mathrm{~cm}^{3} / г$. Масса образца определялась на весах BP 211 D с точностью до $10^{-5}$ г. Образец был ориентирован относительно внешнего магнитного поля с учетом замечаний, высказанных в [4], что позволило 


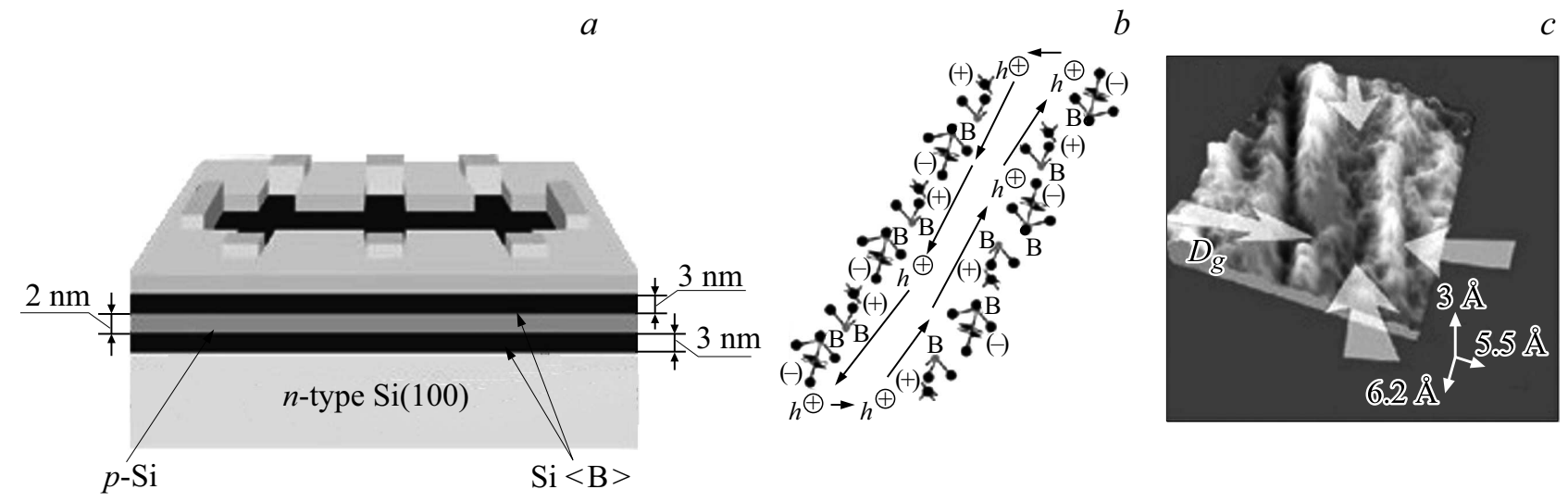

Рис. 1. $a$ - схематическое изображение многотерминальной сандвич-структуры. $b, c-$ модель и трехмерное полученное методом сканирующей туннельной микроскопии изображение фрагмента краевого канала, ограниченного барьерами, состоящими из дипольных центров бора с отрицательной корреляционной энергией, упорядоченных обменным взаимодействием через одиночные дырки.

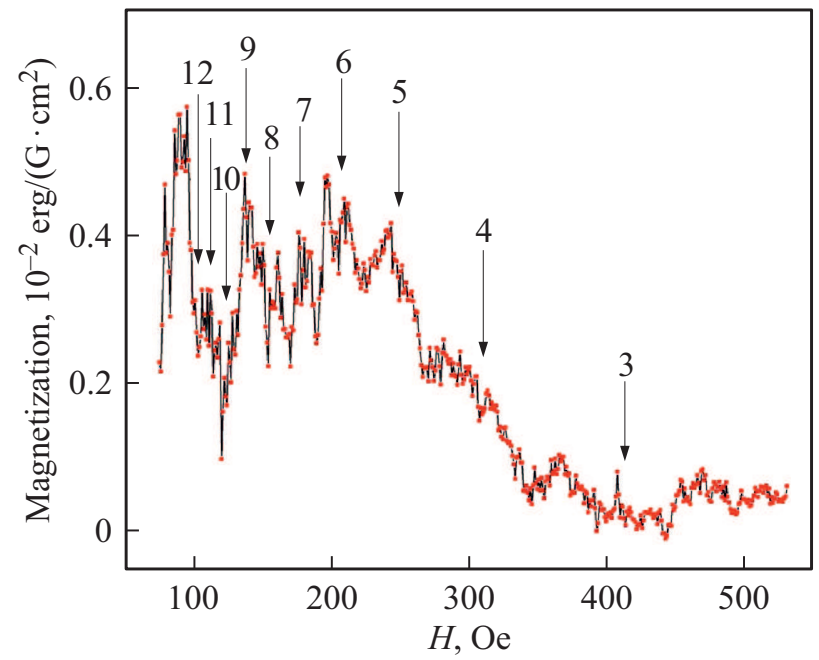

Рис. 2. Полевая зависимость намагниченности кремниевого наносандвича, показывающая эффект де Гааза-ван Альфена при комнатной температуре в слабых магнитных полях. Указаны значения фактора заполнения.

избежать „размытия“ осцилляций из-за усреднения магнитного поля на линейном размере структуры.

Особо отметим, что образец, на котором проводились измерения, не подвергался предварительно каким-либо внешним воздействиям, включая оптические, термические или со стороны электрического или магнитного полей.

Полевая зависимость намагниченности, представленная на рис. 2, содержит хорошо разрешенные осцилляции де Гааза-ван Альфена до значений фактора заполнения $v=12$ включительно. Полученная зависимость характеризуют низкоразмерную систему, проходящую в своем развитии через последовательность термодинамически равновесных состояний, реализуемых путем пошагового изменения внешнего магнитного поля. Измеряе- мый сигнал регистрировался при нахождении системы в состоянии термодинамического равновесия. Это дает основания провести анализ наблюдаемых в эксперименте осцилляций в рамках равновесной термодинамики.

\section{3. Плотность состояний на уровне Ферми}

В равновесной термодинамике свободная энергия Гельмгольца задается выражением

$$
G=P V-T S-M H+\mu N,
$$

где $M-$ магнитный момент образца, $\mu-$ химический потенциал, $N$ - число частиц, и с учетом $B \approx H$.

Отсюда

$$
\begin{gathered}
M=-\left(\frac{\partial G}{\partial H}\right)_{P, T, \mu}, \quad N=\left(\frac{\partial G}{\partial \mu}\right)_{P, T, H}, \\
\frac{\partial M}{\partial \mu}=-\frac{\partial}{\partial \mu} \frac{\partial G}{\partial H}, \\
\frac{\partial N}{\partial H}=\frac{\partial}{\partial H} \frac{\partial G}{\partial \mu} \Rightarrow\left(\frac{\partial M}{\partial \mu}\right)_{H}=\left(\frac{\partial N}{\partial H}\right)_{\mu} .
\end{gathered}
$$

Используя соотношение Максвелла и линейную зависимость вырождения $g$ от поля, приходим к соотношению

$$
\frac{\Delta M}{N}=\frac{\Delta \mu}{H^{*}},
$$

где $\Delta M-$ размах осцилляции магнитного момента, $H^{*}$ - критическое значение поля,

$$
N=p_{2 \mathrm{D}} S,
$$

$p_{2 \mathrm{D}}$ - двумерная плотность дырок.

Положим $\Delta \mu \approx \Delta W-$ эффективная энергетическая щель. Тогда

$$
\Delta W=\frac{H^{*} \Delta M}{N} .
$$


С изменением магнитного поля на $\Delta H$ число состояний, прошедших через уровень Ферми, определяется как $v \Delta g$. Следовательно, остаточное число состояний в щели между уровнями Ландау есть

$$
N_{g}=v \frac{\Delta H}{H^{*}} g=N \frac{\Delta H}{H^{*}} .
$$

Под $\Delta H$ мы будем понимать расстояние в магнитном поле между локальными максимумом и минимумом осцилляций намагниченности при целочисленных факторах заполнения, различающихся на единицу.

Если $D_{g}-$ средняя двумерная плотность состояний (DOS) на уровне Ферми в интервале магнитных полей $\Delta H$, то

$$
N_{g}=D_{g} \Delta W .
$$

Выразим из уравнения (3) двумерную плотность состояний и преобразуем следующим образом с учетом (1) и (2):

$$
D_{g}=\frac{N_{g}}{\Delta W}=\frac{N \Delta H}{H^{*}} \frac{N}{H^{*} \Delta M}=\frac{N^{2} \Delta H}{H^{* 2} \Delta M}=\frac{p_{2 \mathrm{D}}^{2} S^{2} \Delta H}{H^{* 2} \Delta M} .
$$

Выражение позволяет оценить среднюю DOS на уровне Ферми в интервале $\Delta H$, т.е. $D_{g}$ определяет значение DOS между верхним занятым и нижним незаселенным уровнями Ландау.

\section{4. Выбор расчетной модели и вычисление плотности состояний}

При низких температурах и в больших магнитных полях плотность состояний в пространственно ограниченной двумерной электронной системе характеризуется пиками в окрестности уровней Ландау и малыми значениями в каждой из энергетических щелей. Для описания эксперимента используются различные модели плотности состояний $[5,6]$, которые различаются выбором функции статистического распределения.

Анализируя результаты, полученные при измерении удельной теплоемкости пленок GaAs-GaAlAs, авторы [5] показали, что наилучшим приближением является использование гауссовой плотности состояний с плоским базовым фоном. Повышение температуры приводит к уширению гауссова распределения и появлению заметного вклада состояний между уровнями Ландау в полную плотность состояний, что соответствует теоретическим предсказаниям [7] и подтверждено в экспериментах по измерению удельной теплоемкости носителей в пленках GaAs-GaAlAs [5].

С другой стороны, совершенно очевидно, что основное различие в соответствии той или иной функции распределения плотности состояний экспериментальным зависимостям проявляется только в сильных магнитных полях, в то время как в слабых магнитных полях, что имеет место в нашем случае, это различие не выявляется.

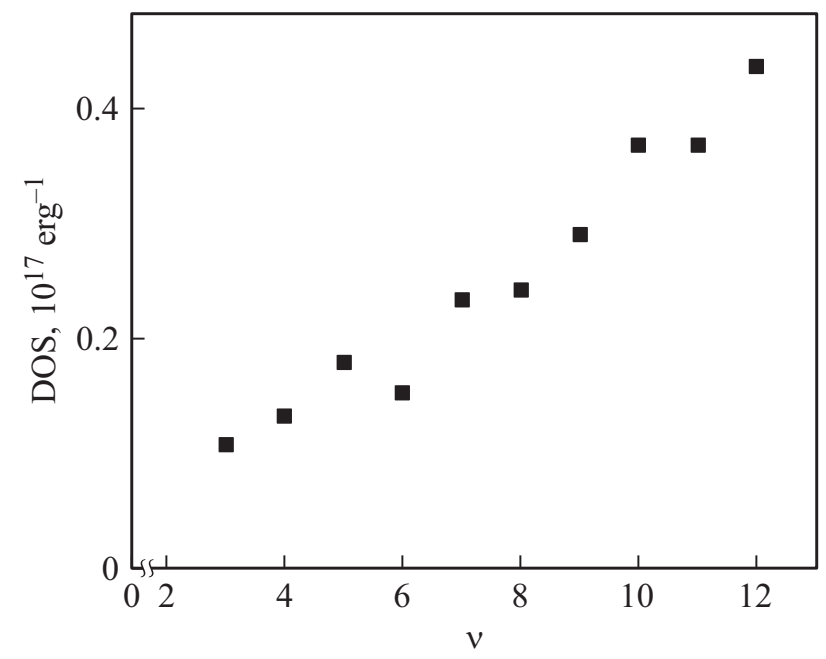

Рис. 3. Зависимость плотности состояний в кремниевом наносандвиче от фактора заполнения при комнатной температуре.

Поэтому мы оцениваем плотность состояний между уровнями Ландау (на уровне Ферми), основываясь на простой модели, предложенной Андо и Уэмурой [6], в которой DOS между соседними уровнями Ландау принимается постоянной.

Следуя [8], будем считать, что „ширина“ $\Delta H$ осцилляций намагниченности при целочисленных факторах заполнения пропорциональна числу состояний в щели.

Например, для осцилляции, наблюдаемой в поле $H^{*}=414 \ni \quad(v=3)$, имеем $\Delta H \approx 6 \ni$, $\Delta M \approx 2.7 \cdot 10^{-6}$ эрг/Гс. Тогда получаем

$$
D_{g}=\frac{9 \cdot 10^{18} \cdot\left(0.94 \cdot 10^{-2}\right)^{2} \cdot 6}{(414)^{2} \cdot 2.7 \cdot 10^{-6}}=1.03 \cdot 10^{16} \text { эрг }^{-1} \text {. }
$$

Плотность состояний на уровне Ферми для ряда целочисленных факторов заполнения и ее зависимость от фактора заполнения приведены на рис. 3 .

\section{5. Результаты и выводы}

Во всех физических свойствах структуры, исследуемых через транспортные эффекты, пиннинг уровня Ферми имеет место в областях локализованных состояний, которые не проводят тока [9]. Однако для термодинамически равновесных свойств структуры, таких как удельная теплоемкость или намагниченность, различие между локализованными и нелокализованными состояниями не имеет значения [5]. Поэтому равновесные характеристики показывают полную плотность состояний и по осциллирующим зависимостям намагниченности можно исследовать термодинамическую плотность состояний в магнитном поле, являющуюся интегральной характеристикой энергетического спектра структуры.

Токоведущие краевые состояния вводятся как пересечения уровней Ландау и уровня Ферми у краев образца [10]. Таким образом, полное число краевых состояний 
равно фактору заполнения - числу заполненных уровней Ландау.

В теоретических исследованиях не было уделено особого внимания зависимости значения DOS между уровнями Ландау от магнитного поля и фактора заполнения. Однако в работе [11] было предсказано сильное уменьшение DOS между уровнями Ландау с магнитным полем в предположении пространственных корреляций случайного потенциала.

В экспериментальном исследовании [12], выполненном на двумерной структуре, сформированной в модулированно-легированном гетеропереходе $\mathrm{AlGaAs} / \mathrm{GaAs}$, при низких температурах и магнитных полях до 15 Тл, было показано, что DOS между уровнями Ландау линейно возрастает с фактором заполнения.

Мы оределили зависимость DOS от фактора заполнения при комнатной температуре (рис. 3), основываясь на модели плотности состояний с энергетически независимой основой $[6,8]$, которая показывает рост плотности состояний на уровне Ферми как функции фактора заполнения, т.е. хорошо коррелирует с результатами, полученным в [12]. Наблюдаемый наклон (рис. 3), повидимому, не может быть связан с перекрытием уровней Ландау или температурным размытием уровня Ферми, что привело бы к невозможности разрешить спектр.

Аналогично $[1,3,12]$ мы полагаем, что экспериментально установленное поведение фоновой DOS, линейно возрастающей с фактором заполнения, отражает непосредственно вклад краевых состояний. Этот вывод согласуется с хорошо установленным представлением, что краевые состояния играют решающую роль в свойствах двумерной структуры в режиме квантового эффекта Холла [10].

\section{Конфликт интересов}

Авторы заявляют об отсутствии у них конфликта интересов.

\section{Список литературы}

[1] N.T. Bagraev, V.Yu. Grigoryev, L.E. Klyachkin, A.M. Malyarenko, V.A. Mashkov, V.V. Romanov. Semiconductors, 50 (8), 1025 (2016).

[2] N.T. Bagraev, L.E. Klyachkin, V.V. Romanov, A.I. Ryskin. Low Temp. Phys., 40 (4), 352 (2014).

[3] N.T. Bagraev, V.Yu. Grigoryev, L.E. Klyachkin, A.M. Malyarenko, V.A. Mashkov, V.V. Romanov, N.I. Rul'. Low Temp. Phys., 43 (1), 1 (2017).

[4] D. Shoenberg. Magnetic oscillations in metals (Cambridge University Press, Cambridge 1984).

[5] E. Gornik, R. Lassing, G. Strasser, H.L. Stormer, A.C. Gossard, W. Wiegmann. Phys. Rev. Lett., 54, 1820 (1985).

[6] T. Ando, Y. Uemura. J. Phys. Soc. Japan, 37, 1044 (1974).

[7] W. Zawadzki, R. Lassnig. Solid State Commun., 50, 537 (1984).
[8] S.A.J. Wiegers, M. Specht, L.P. Levy, M.Y. Simmons, D.A. Ritchie, A. Cavanna, B. Etienne, G. Martinez, P. Wyder. Phys. Rev. Lett., 79, 3238 (1997).

[9] K. von Klitzing, G. Ebert. Physica (Utrecht), 117B\&118B, 682 (1983).

[10] B.I. Halperin. Phys. Rev. B, 25, 2185 (1982).

[11] L. Spies, W. Apel, B. Kramer. Phys. Rev. B, 55, 4057 (1997).

[12] M.P. Schwarz, M.A. Wilde, S. Groth, D. Grundler, Ch. Heyn, D. Heitmann. Phys. Rev. B, 65, 245315 (2002).

Редактор Л.В. Шаронова

\section{Thermodynamic description of oscillations of silicon nanostructure magnetization in weak magnetic fields at the room temperature. Density of states}

\author{
V.V. Romanov ${ }^{1}$, V.A. Kozhevnikov ${ }^{1}$, N.T. Bagraev ${ }^{\mathbf{1 , 2}}$ \\ ${ }^{1}$ Peter the Great St. Petersburg Polytechnic University, \\ 195251 St. Petersburg, Russia \\ 2 loffe Institute, \\ 194021 St. Petersburg, Russia
}

Abstract Observation of the de Haas-van Alphen oscillations in the studies of silicon nanostructure at the room temperature in weak magnetic fields does possible use of thermodynamic ratios for calculation of density of states at the Fermi level at critical values of the external magnetic field for integer factors of filling. 